\title{
Bilateral Episcleral Corkscrew Vessels: Expedition into the Unknown - Case Report
}

\author{
Carolina Madeira ${ }^{a} \quad$ Gonçalo Alves ${ }^{b} \quad G$ Gonçalo Godinho ${ }^{a} \quad$ Jorge Meira ${ }^{a}$ \\ Vítor Leal $^{a}$ Manuel Falcão ${ }^{a, c}$ Fernando Falcão-Reis ${ }^{a, c}$ \\ João Barbosa Breda a, d, e \\ aDepartment of Ophthalmology, Centro Hospitalar e Universitário de São João, Porto, \\ Portugal; ${ }^{b}$ Department of Neuroradiology, Centro Hospitalar e Universitário de São João, \\ Porto, Portugal; ' ${ }^{2}$ epartment of Surgery and Physiology, Faculty of Medicine of University \\ of Porto, Porto, Portugal; ${ }^{\mathrm{d} C a r d i o v a s c u l a r ~ R \& D ~ C e n t e r, ~ F a c u l t y ~ o f ~ M e d i c i n e ~ o f ~ t h e ~ U n i v e r s i t y ~}$

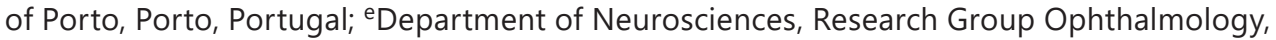 \\ $\mathrm{KU}$ Leuven, Leuven, Belgium
}

Keywords

Episcleral venous pressure - Ocular hypertension - Idiopathic · Case report

\begin{abstract}
We aim to report a case of a middle-aged woman with bilateral idiopathic elevated episcleral venous pressure (IEEVP) and its difficulties in the diagnostic approach. Particularly in this case, the atypical feature of ocular hypertension without glaucomatous optic nerve damage may be misleading. We present a 66-year-old woman with longstanding bilateral "red eyes." Clinical findings included bilateral episcleral vessel engorgement and tortuosity and raised intraocular pressure with open iridocorneal angles. Despite ocular hypertension, glaucomatous neuropathy was absent and confirmed by normal structural (optical coherence tomography) and functional (standard automated perimetry) tests. The systemic workup was unremarkable. Magnetic resonance angiography showed bilateral dilated superior ophthalmic veins. Cerebral digital subtraction angiography was requested, and no carotid-cavernous fistula (or other significant vascular findings) was identified. The diagnosis of IEEVP was assumed. In conclusion, our case highlights the systematic investigation necessary in cases of bilateral episcleral vessel engorgement and tortuosity and the possible differential diagnosis to be considered to rule out life-threatening causes of elevated episcleral venous pressure. It is important for clinicians to be aware of IEEVP even in patients with atypical features that despite significative ocular hypertension had no glaucomatous damage.
\end{abstract}




\section{Case Reports in Ophthalmology}

\section{Background}

Elevation of episcleral venous pressure (EEVP) causes aqueous outflow reduction, and consequently elevated intraocular pressure, which can lead to glaucomatous optic nerve damage if left untreated [1]. Several pathologies can cause EEVP, including arteriovenous malformations, such as carotid-cavernous fistulas, orbital varices, and Sturge-Weber syndrome. Other conditions that lead to venous obstruction include retrobulbar tumours, cavernous sinus thrombosis, thyroid orbitopathy, and superior vena cava syndrome [1,2]. Idiopathic EEVP (IEEVP) or RadiusMaumenee syndrome is a rare entity and should be considered a diagnosis of exclusion [3-5].

IEEVP can occur as a unilateral or a bilateral condition at any age, with no gender preference $[6,7]$. Clinically, IEEVP leads to dilated episcleral veins, intraocular hypertension with an open iridocorneal angle, visible blood in Schlemm's canal, and optic nerve damage [1]. We report a case of a middle-aged woman with bilateral IEEVP and no glaucomatous damage and its diagnostic approach.

\section{Case Presentation}

A 66-year-old woman presented to our outpatient clinic with chronic asymptomatic bilateral conjunctival hyperaemia for over a year, which was unresponsive to medical treatment. Her medical history included a history of treated arterial hypertension, dyslipidaemia, atrial fibrillation, and type 2 diabetes mellitus. At presentation, best-corrected visual acuity was $20 / 20$ in both eyes (OU). The presence of bilateral episcleral corkscrew vessels was notorious at biomicroscopy (Fig. 1). No other ocular surface changes were noted. Intraocular pressure (IOP) measured by Goldmann applanation tonometry was 27 and $28 \mathrm{~mm} \mathrm{Hg}$ in the right (OD) and left (OS) eye, respectively. Corneal pachymetry was 501 and $499 \mu \mathrm{m}$ in the OD and OS, respectively. No proptosis was observed. Ocular motility was normal, and the pupils were isocoric with normal light reflexes. Gonioscopy revealed an open angle, with blood in the Schlemm's canal in the OD. The ocular fundus was unremarkable, with no vascular abnormalities and a cup-to-disc ratio of approximately $0.2 \mathrm{H} \times 0.2 \mathrm{~V}$ in the $\mathrm{OD}$ and $0.1 \mathrm{H} \times 0.1 \mathrm{~V}$ in the $\mathrm{OS}$ (Fig. 2). No carotid or ocular bruits were detected.

The patient had no family history of glaucoma nor a history of head or neck trauma. No symptoms of headache, diplopia, pulsatile tinnitus, and pulsation of the orbit were present.

Optical coherence tomography (OCT; Spectralis; Heidelberg Engineering) revealed a retinal nerve fibre layer average thickness of 87 and $84 \mu \mathrm{m}$ in the OD and OS, respectively. Bruch's membrane opening minimum rim width and the macular ganglionar cell layer analyses
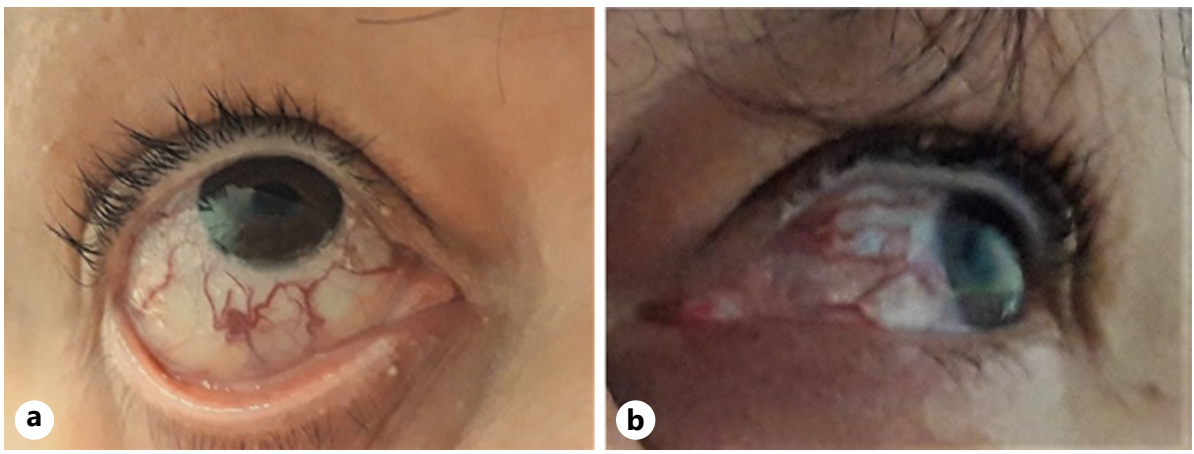

Fig. 1. Anterior segment photos of the right (a) and left (b) eyes showing episcleral vasculature engorgement and tortuosity. 


\section{Case Reports in Ophthalmology}
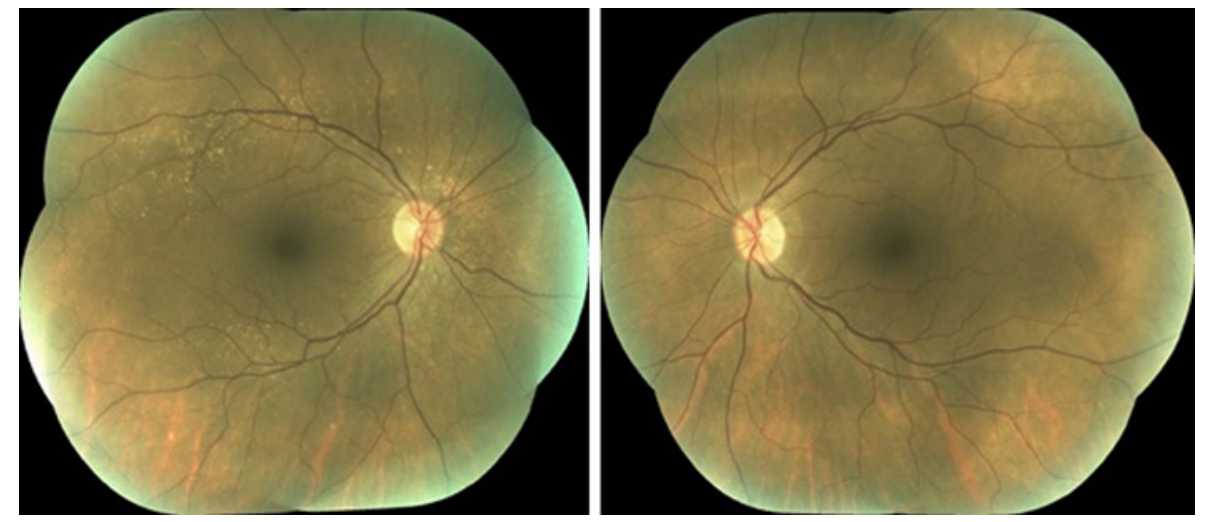

Fig. 2. Fundus photography showing a normal optic disc, without cupping, no vascular tortuosity, and perivascular miliar drusen. Visual field testing and optic nerve OCT were normal.
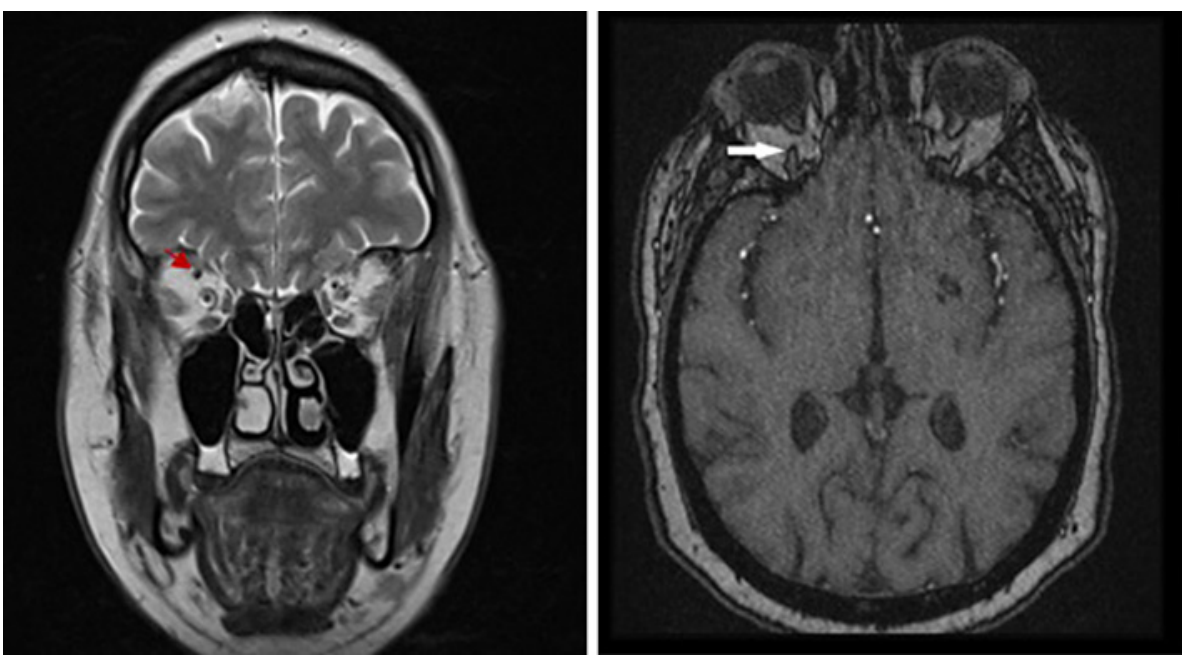

Fig. 3. Brain MRI. Coronal T2 turbo spin echo and TOF MRA showing enlargement of the superior ophthalmic vein on the right ( $4 \mathrm{~mm}$ of diameter) (arrow). Normal appearance of the orbital fat. No signs of proptosis. Cavernous sinus unremarkable (not shown).

were within normal limits in OU. Visual field testing (Standard automated perimetry Humphrey Field Analyzer; Zeiss; 24:2 SITA Standard) was within normal limits. As such, the patient was diagnosed with ocular hypertension related to EEVP. IOP-lowering drops (timolol $5 \mathrm{mg} / \mathrm{mL}$ b.i.d) were initiated, while a diagnostic workup was performed to identify potential causes of EEVP.

The brain and orbital CT scans performed were within normal limits, which excluded hypertrophy of the extraocular muscles, orbital, or intracranial space-occupying lesions. Thyroid function and thyroid antibody tests were within normal limits. A workup for vascularrelated pathologies (e.g., carotid-cavernous fistula and cavernous sinus thrombosis) was performed. Magnetic resonance angiography showed bilateral dilated superior ophthalmic veins (Fig. 3). Cerebral digital subtraction angiography was negative for carotid-cavernous fistula (Fig. 4). Thoracic CT angiography scans revealed stigma of mild pulmonary hypertension and no venous cava obstruction.

The diagnosis of IEEVP was assumed. The initial IOP-lowering treatment (timolol $5 \mathrm{mg} / \mathrm{mL}$ ) reduced the IOP to $22 \mathrm{~mm} \mathrm{Hg}$ bilaterally. Nonetheless, after 3 months, despite good patient compliance, the IOP rose to 28 and $32 \mathrm{~mm} \mathrm{Hg}$ in the OD and OS, respectively. A second drug 


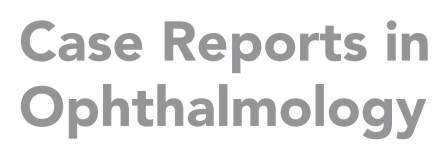

Case Rep Ophthalmol 2022;13:109-115

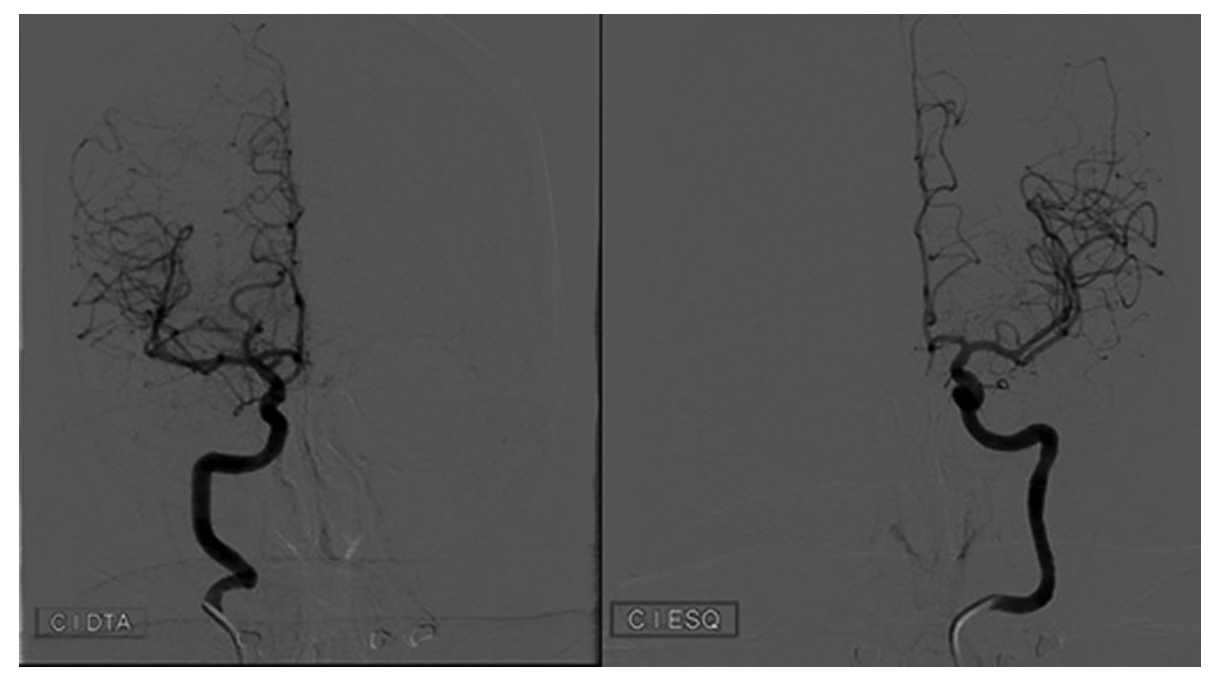

Fig. 4. Digital subtraction angiography was negative for carotid-cavernous fistula or other significant vascular abnormalities.

(latanoprost $50 \mu \mathrm{g} / \mathrm{mL}$ ) was added, and good IOP control was achieved, with intraocular pressure values of $17 \mathrm{~mm} \mathrm{Hg} \mathrm{OU.}$

\section{Discussion and Conclusion}

This clinical case highlights the possible differentials to be considered when faced with a case of ocular hypertension (with or without glaucomatous neuropathy) with engorged episcleral vessels. We summarize the systematic investigations to be done while ruling out the different possible aetiologies $[1,6,7]$. The diagnostic approach of these patients is not straightforward and can be challenging.

Aqueous humour drainage occurs through 2 pathways: conventional and uveoscleral. The episcleral veins are the distal portion of the conventional aqueous humour outflow pathway. Aqueous humour flows into the trabecular meshwork, Schlemm's canal, and collector channels and then through aqueous veins that drain into the episcleral veins [8]. The episcleral venous plexus drains into the anterior ciliary vein and into the superior ophthalmic vein. Subsequently, it flows into the cavernous sinus, proceeding into the superior vena cava through the internal jugular vein, and finally into the right atrium [9]. Any obstruction to this drainage pathway may cause EEVP with consequent episcleral vessel dilation and ocular hypertension [10]. Therefore, the pressure gradient between the anterior chamber and the episcleral veins influences the aqueous outflow through this pathway [2].

Anterior segment OCTA has emerged as a valuable method to study the anterior segment vasculature and show potential to provide new knowledge about the role of the episcleral vessels in intraocular pressure regulation. Recently, Zhao et al. [11] analysed the episcleral vasculature in Sturge-Weber syndrome patients with juvenile-onset glaucoma through OCTA. The vessel density and diameter index correlated with the IOP increase and the cup-disc ratio, indicating that episcleral vascular hypertrophy may be a risk factor for glaucoma development in these cases [11].

The obstruction of the aqueous drainage pathway could be caused by arteriovenous malformations/fistulas or venous obstruction. Carotid-cavernous fistulas are the most common cause of EEVP. Other causes include thyroid orbitopathy, Sturge-Weber syndrome, orbital

\section{Karger'}


tumours or varices, venous sinus thrombosis, superior vena cava syndrome, and obstruction of the pulmonary or jugular veins $[1,2,12]$

IEEVP or Radius-Maumenee syndrome is characterized by arterialized, engorged, and tortuous episcleral veins, with raised IOP, and, almost always, open-angle glaucoma is already present at the time of diagnosis $[3,6,7,13]$. It affects individuals of any age with no gender preference. This condition could be unilateral or bilateral; when bilateral, it may be asymmetrical [3]. IEEVP is a diagnosis of exclusion and can only be established after other conditions, some of which are life-threatening, have been excluded [1].

Typically, in IEEVP, no dilation of superior ophthalmic veins or reversal of blood flow is observed, although some cases of IEEVP with these features have been published [1,7]. In our case, bilateral dilated superior ophthalmic veins were present, leading to a high suspicion of a carotid-cavernous fistula, which was not confirmed by the cerebral digital subtraction angiography. Dural arteriovenous shunts or fistulas or indirect cavernous sinus fistula are the most common missed diagnoses when IEEVP is suspected, as the flow through this fistula is low rendering its detection extremely hard by neuro-radiologic examination $[3,7]$. However, patients with indirect cavernous sinus fistula usually present with symptoms and signs such as proptosis, conjunctival chemosis, ocular motility disturbance, bruit, decreased visual acuity, headache, and retinal venous congestion. All were absent in our case, which makes the diagnosis of dural arteriovenous shunts or fistulas unlikely.

Moreover, in this case, mild pulmonary hypertension was present. A few cases of extremely severe pulmonary hypertension (usually heritable pulmonary hypertension) leading to EEVP have been reported $[14,15]$. The present case did not have any clinical signs or symptoms of pulmonary hypertension (fatigue, cyanosis, angina, or peripheral oedema), and only mild stigma of the disease was present in CTA, which would not be sufficient to cause clinically relevant EEVP.

Characteristically, the lack of characteristic symptoms of EEVP delays the diagnosis $[7,16]$. As such, patients with a longstanding undiagnosed EEVP present with advanced optic nerve cupping and pronounced visual field loss $[1,12,13,16]$. In our case, no structural or functional glaucomatous damage was found, which most likely means that this patient was diagnosed at a relatively early course of the disease.

Because no clear aetiology has yet been identified for IEEVP, its treatment is similar to primary open-angle glaucoma $[3,5,17]$. Nonetheless, IEEVP has lower responsiveness to medical treatment $[3,5]$. In our case, the patient was initially treated with timolol, which allowed a good IOP control. However, 3 months later, the IOP increased, requiring the addition of a prostaglandin, and consequently, an IOP of $17 \mathrm{~mm} \mathrm{Hg}$ in OU was achieved. This illustrates the progressive increase of IOP in IEEVP patients. Moreover, the majority of patients may eventually require surgery.

The majority of the cases requiring surgery underwent trabeculectomy with or without antimetabolites, and, in a few cases, non-penetrating deep sclerectomies have been reported $[4,18]$. Both surgeries allow good IOP control. A major concern in the surgical management of these patients is the high risk of uveal effusion. As such, taking appropriate measures to prevent sudden hypotony and inflammation is imperative to avoid uveal effusion [17]. Preoperative administration of intravenous mannitol, a slow decompression during surgery when entering the globe (consider starting with a decompression paracentesis), prophylactic sclerotomies, anterior chamber maintainers (e.g., cannula with continuous infusion of balanced salt solution or viscoelastic agent), and application of tight adjustable sutures on the scleral flap to decrease the probability of excessive filtering postoperatively may be good options $[17,19,20]$. During the postoperative period, the use of cycloplegic eye drops and oral and topical glucocorticoids to reduce inflammation

\section{Karger'}


may reduce the risk of choroidal effusion $[17,19]$. Another hypothetical alternative to reduce the risk of uveal effusion would be a surgical procedure with a less abrupt IOP reduction such as bleb-forming minimally invasive glaucoma surgeries (MIGS). Since a trabecular approach is not suitable (since this route is dependent on EVP), the only potential options are the bleb-forming MIGS.

Overall, after surgery, patients achieve good IOP control. However, previous reports described that the dilated episcleral vessels did not regress, and there was a maintenance of blood in Schlemm's canal after surgery [3].

In conclusion, we present a case of IEEVP, with an atypical scenario since a bilateral superior ophthalmic vein dilatation was present and no glaucomatous damage was observed. We summarize the diagnostic approach to a patient with EEVP and highlight the relevance of excluding life-threatening conditions.

\section{Acknowledgments}

This manuscript does not include any non-author contributors to acknowledge.

\section{Statement of Ethics}

Written informed consent was obtained from the patient for publication of this case report and any accompanying images. Ethical approval from the local committee has been obtained.

\section{Conflict of Interest Statement}

The authors have no conflicts of interest to declare.

\section{Funding Sources}

This manuscript did not receive any funding.

\section{Author Contributions}

All authors attest that they meet the current criteria for authorship. C.M., G.G., J.M., V.L., and J.B.B. analysed and interpreted all clinical data. G.A. collected and analysed CT scans, MRI, and cerebral digital subtraction. M.F. and F.F.-R. reviewed the manuscript. All authors read and approved the final manuscript.

\section{Data Availability Statement}

The authors confirm that the data supporting the findings of this study are available within the article. 


\section{References}

1 Greslechner R, Oberacher-Velten I. [Glaucoma due to elevated episcleral venous pressure]. Ophthalmologe. 2019 May; 116(5):423-9.

2 European Glaucoma Society terminology and guidelines for glaucoma, 4th edition - chapter 2: classification and terminologysupported by the EGS Foundation: part 1: foreword; introduction; glossary; chapter 2 classification and terminology. Br J Ophthalmol. 2017 May;101(5):73-127.

3 Rong X, Li M. Advanced glaucoma secondary to bilateral idiopathic dilated episcleral veins - a case report. BMC Ophthalmol. 2018 Aug 23;18(1):207.

4 Sun CQ, Medert CM, Chang TC. Idiopathic elevated episcleral venous pressure in a teenager. Am J Ophthalmol Case Rep. 2020 Jun;18:100712.

5 Usha Tejaswini S, Sivakumar P, Upadhyaya S, Venkatesh R. Elevated episcleral venous pressure and its implications: a case of Radius-Maumenee syndrome. Indian J Ophthalmol. 2020 Aug;68(8):1683-5.

6 Radius RL, Maumenee AE. Dilated episcleral vessels and open-angle glaucoma. Am J Ophthalmol. 1978 Jul; 86(1):31-5.

7 Rhee DJ, Gupta M, Moncavage MB, Moster ML, Moster MR. Idiopathic elevated episcleral venous pressure and open-angle glaucoma. Br J Ophthalmol. 2009 Feb;93(2):231-4.

8 Fautsch MP, Johnson DH. Aqueous humor outflow: what do we know? Where will it lead us? Invest Ophthalmol Vis Sci. 2006 Oct;47(10):4181-7.

9 Lee SS, Robinson MR, Weinreb RN. Episcleral venous pressure and the ocular hypotensive effects of topical and intracameral prostaglandin analogs. J Glaucoma. 2019 Sep;28(9):846-57.

10 European Glaucoma Society terminology and guidelines for glaucoma, 4th edition - chapter 2: classification and terminologysupported by the EGS Foundation: part 1: foreword; introduction; glossary; chapter 2 classification and terminology. Br J Ophthalmol. 2017;101(5):73-127.

11 Zhao Z, Xu L, Ding X, Wu Y, Zhu X, Fu Y, et al. Optical coherence tomography angiography of perilimbal vasculature in Port-Wine stain and Sturge-Weber Syndrome patients. Invest Ophthalmol Vis Sci. 2020;61(4):43.

12 Moster M. Episcleral venous pressure and glaucoma. J Curr Glaucoma Pract. 2009;3:5-8.

13 Parikh RS, Desai S, Kothari K. Dilated episcleral veins with secondary open angle glaucoma. Indian J Ophthalmol. 2011 Mar;59(2):153-5.

14 Faure C, Miocque S, Fleury L, Robert MP. Primary pulmonary arterial hypertension diagnosed via its ophthalmic features in an adult: diagnosis and therapeutic challenges. Br J Ophthalmol. 2012 Mar;96(3):459-69.

15 Watanabe M, Makino S, Obata H. Bilaterally dilated episcleral vessels in patients with heritable pulmonary arterial hypertension. J Gen Fam Med. 2017;18(6):464-5.

16 Marques SHM, Farinha C, Martins A, Faria P. Radius-Maumenee syndrome: a rare cause of glaucoma. BMJ Case Rep. 2018 Jan 5;2018:bcr2017223255.

17 Pradhan ZS, Kuruvilla A, Jacob P. Surgical management of glaucoma secondary to idiopathic elevated episcleral venous pressure. Oman J Ophthalmol. 2015 May-Aug;8(2):120-1.

18 Güven D, Karakurt A, Ziraman I, Hasiripi H. Non-penetrating deep sclerectomy in unilateral open-angle glaucoma secondary to idiopathic dilated episcleral veins. Eur J Ophthalmol. 2002 Jan-Feb;12(1):66-8.

19 Bakir B, Pasquale LR. Causes and treatment of choroidal effusion after glaucoma surgery. Semin Ophthalmol. 2014 Sep-Nov;29(5-6):409-13.

20 Schrieber C, Liu Y. Choroidal effusions after glaucoma surgery. Curr Opin Ophthalmol. 2015 Mar;26(2):134-42. 